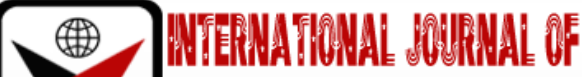

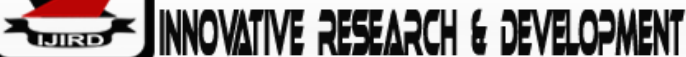

ISSN 2278-0211 (Online)

\section{Case of Covid-19 Presenting with Common Personal Nerve Mononeuropraxia}

\author{
Dr. Mazaher Hassan Jaffer \\ Ph.D. Student, School of Pure and Applied Sciences, \\ Mount Kenya University, Kenya \\ Dr. Mohamed Gaman \\ Consultant Diabetologist, The Comprehensive Diabetes Care Center, Kenya \\ Dr. Mary Muriuki \\ Dean, School of Pure and Applied Sciences, \\ Mount Kenya University, Kenya
}

\begin{abstract}
:
Introduction: Common peroneal nerve palsy presents with a foot drop and weakness of foot eversion(Stewart, 2008). It has always been associated with mechanical causes, with very few cases being bilateral which were also assumed to be mechanical but secondary to mechanical deformities or compression related causes such as tumors(Ag Lamat et al., 2020) or severe weight loss due to anorexia nervosa(Kershenbaum et al., 1996).

COVID-19 is a disease, since it's onset in December 2019 that is relatively new and manifesting in different ways. The variation of symptoms related to COVID-19 involves the upper and lower respiratory, gastrointestinal, cardiovascular, as well as nervous system. Cases have been reported with post infectious GuillainBarre syndrome, olfactory neuropraxia or neuritis, and even encephalitis (Montalvan et al., 2020). There has not been a prior documented case reported with footdrop due to COVID-19 or any other infectious cause.

Methods: This case reported is of a 45-year-olddiabetic and hypothyroid male of Indian origin who was diagnosed to have symptomatic COVID-19 by PCR of his nasopharyngeal swab with respiratory distress requiring supplemental oxygen for the first 10 days and anosmia for about 2 weeks, after which he developed an acute footdrop on the rightside effecting eversion of the foot and sensation in the dorsal aspect of the foot effecting more of the fine touch and two-point differentiation. He was initiated on steroids, nebulized ipratropium bromide, salbutamol, and beclomethasone as well as dual antiplatelet therapy with a factor X inhibitor during the first 10 days of treatment. As he stabilized, he was asked to continue antiplatelet and factor X inhibition therapy with 90mg of cerebroprotein once a day for one month even after turning negative through PCR from the nasopharynx to COVID, and his footdrop improved over a period of 3 months subjectively about $70 \%$ but still persists.

Conclusion:As cases unfold with COVID-19due to its unusual induction of the human immune response, mononeuritis involving the olfactory nerve is a common phenomenon(Kirschenbaum et al., 2020) but the possibility of other forms of mononeuritis may also be a possible presentation as found in this case. The presentation remains to be more of a neuropraxia because it seems to be improving with time since its onset after 4 weeks and may resolve fully
\end{abstract}

Keywords: COVID-19, peripheral nervous system, neuropraxia, bilateral foot drop

\section{Introduction}

A confirmed case of COVID-19 in a 45-year-old male who was known to have hypothyroidism, and diabetes which were relatively poorly controlled in the past but was achieving better control of late. His chronic medication taken daily was vildagliptin 50 twice a day, metformin $1000 \mathrm{mg}$ twice a day, Diamicron $30 \mathrm{mg}$ once a day, and thyroxine $100 \mathrm{mcg}$ once a day,

His symptoms experienced included initially rhinorrhea, and general body malaise with worse pain on movement over his loins bilaterally and his trapezius muscles. This lasted 3 days after which he developed a fever of 38.5 degrees Celsius with a cough and chest tightness with audible wheezing. He already commenced on acetaminophen 1 gram three times a day, and at this time he tested for COVID-19 through a nasopharyngeal PCR and was confirmed to be positive for the same.

On presentation (Day1) he seemed weak and agitated with a Glasgow Comma Scale of 15/15. He experienced pain on rotation and flexion as well as extension of his neck. His random sugar levels were at 7.5 mmol/l.His blood pressures were at 90/69 mmhg, with a regular pulse of $112 \mathrm{bpm}$. He was afebrile, displayed nasal hypertrophied and hyperemic turbinates, and tender frontal sinuses. His chest auscultation revealed an episodic wheeze, with oxygen saturations of 


\section{5\%on room air.}

His blood tests done at this time revealed a hemogram without neutrophilia or lymphocytosis, with a mildly elevated CRP of $12.22 \mathrm{ng} / \mathrm{mlborderline} \mathrm{high} \mathrm{TSH}$ of $8.06 \mathrm{uIU} / \mathrm{ml}$, a slightly reduced potassium of $3.3 \mathrm{mmol} / \mathrm{l}$. and a glycosylated Hemoglobin level of $8 \%$ with a slight vitamin D deficiency of $26.76 \mathrm{ng} / \mathrm{ml}$, while his D-dimers with lipid profile were well within normal limits. (Table 1 )

He isolated himself at home as he commenced on Aspirin 75mg once a day, Vitamin D3 at 60,000 iu weekly, mometasone nasal spray and formoterol and budesonide inhaler.

He showed good improvement initially but then on day 3 at home he experienced nocturnal respiratory distress with hypoxia of $85 \%$ saturation for which he was initiated on oxygen supplementation at 5 Liters per minute by nasal prongs at home, while he continued to Isolate himself. He also commenced on nebulization with salbutamol, ipratropium (Combivent) and prednisolone (Pulmicort) solutions. This allowed him to remain comfortable till his next review.

He came for review after 10 days when he was symptomatically feeling better. During this consultation he admitted for the first time to having anosmia with loss of taste, for which he was commenced on a fluticasone nasal spray at $55 \mathrm{mcg}$ once a day bilaterally, and asked him to perform steaming with eucalyptus oil. His chest was clear and he was normotensive at 125/80 mmh, with a pulse of 75 and an oxygen saturation of $95 \%$ on room air as he breathed comfortably. His CRP and D-dimer was elevated so he was commenced on rivaroxaban $20 \mathrm{mg}$ once a day for 10 days that was then reduced to rivaroxaban $10 \mathrm{mg}$ once a day for one month thereafter (Table 1 ).

His symptoms improved till after5 days when he again presented to the clinic having now tested negative for COVID-19 by

PCR from the nasopharynx, but feeling a sudden "heaviness" in the legs bilaterally, more on the right side. This symptom had started acutely the night before while he was in bed and was not associated with any forms of trauma or mechanical compression. The patient did not feel like it was progressive in nature. There was significant pain. His respiratory symptoms had resolved now other than mild exertional dyspnea on severe exertion.

On examination, the patient was normotensive and afebrile with a regular pulse of 90 beats per minute. He had no palpable adenopathy, icterus, cyanosis or pallor. His oxygen saturation was $95 \%$ on room air and he had good air entry bilaterally with a clear chest on auscultation. His pedal pulses were strong and easily palpable bilaterally. His knee jerk and bicep tendon reflexes were normal, with a $5 / 5$ grade of power on movement of all joints except the ankle joint specific to dorsiflexion and eversion of the foot bilaterally which displayed a power of 4/5 and 1/5 on the left and the right side respectively. There was no sign of muscle wasting or fasciculation of the foot or calf muscles. Sensation through double point differentiation and fine touch was preserved in the left foot but absent on the right foot. There was no sign of any other motor or sensoryneuro-deficit, his sense of smell and taste had returned.

His D-dimer had improved and his other blood works had normalized (Table1)

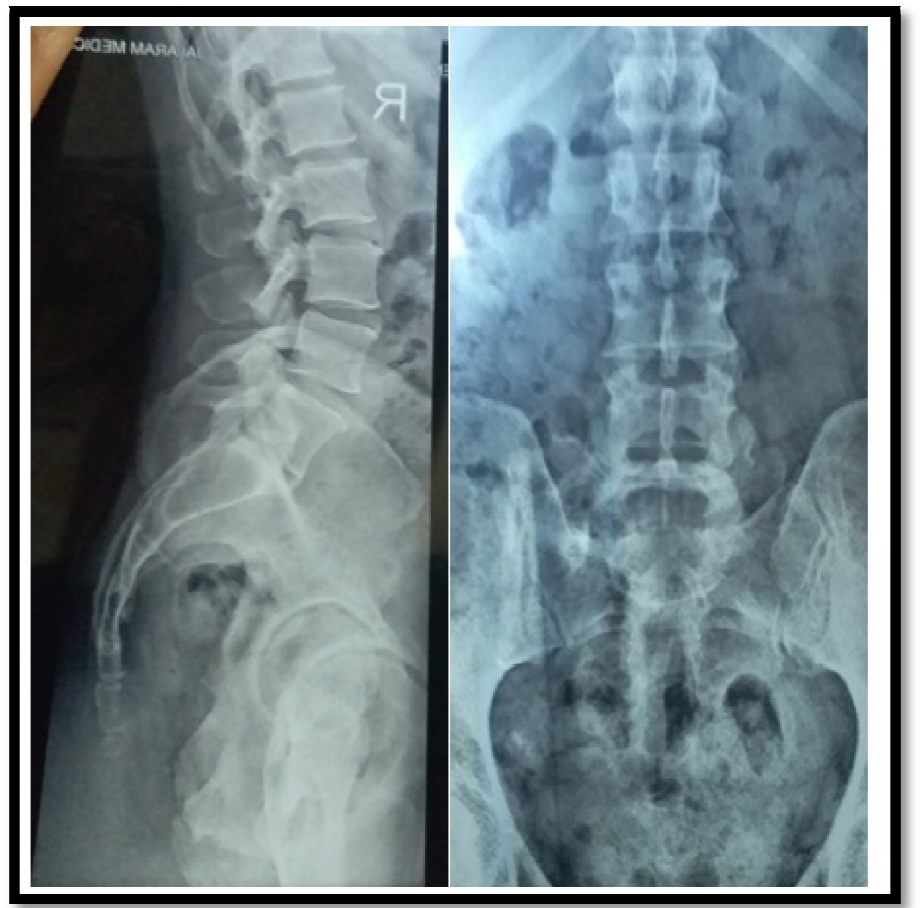

Figure 1: X-Ray of the Lumbar-Sacral

Spine at 5 weeks from the start of illness and 3 weeks since the onset of the foot drop 


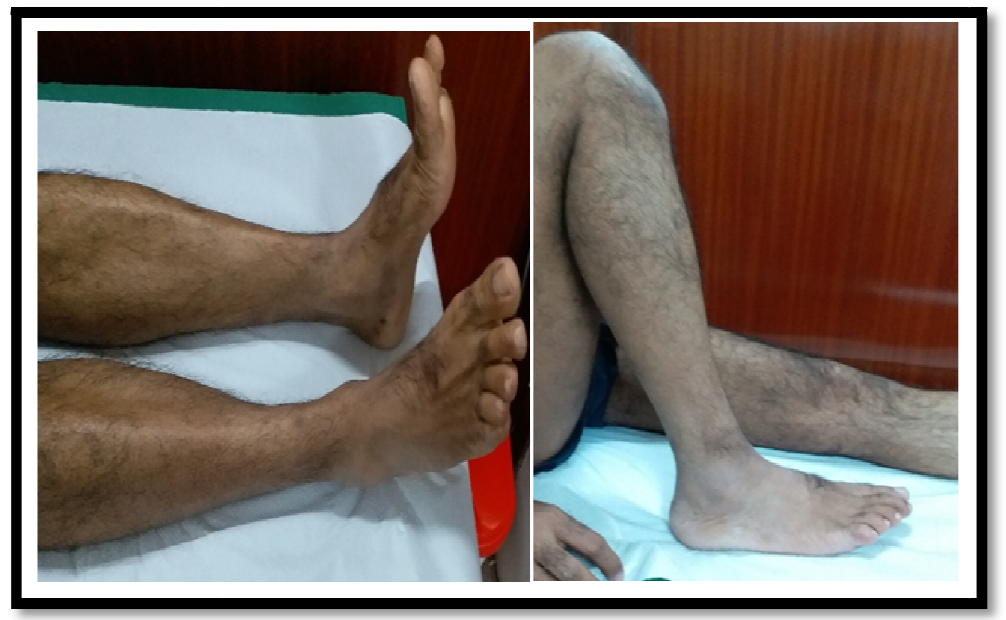

Figure 2: Legs of the Patient in Question as They Continue to Experience Weakness on Dorsiflexion and Eversion

Here the patient has been asked to dorsiflex both feet maximally 2 weeks after the onset of the nerve palsy. There is no sign of muscle wasting or fasciculation.

\begin{tabular}{|c|c|c|c|c|c|c|c|c|c|}
\hline DATE & WBC & $\mathbf{N \%}$ & $\mathbf{L \%}$ & D-dimer & CRP & Creat & NA & K & CL \\
\hline $\begin{array}{c}\text { Ref } \\
\text { Range }\end{array}$ & $\begin{array}{c}\mathbf{4 - 1 1} \mathbf{x} \\
\mathbf{1 0} / \mathbf{L}\end{array}$ & $\begin{array}{c}\mathbf{4 0 - 7 0} \\
\mathbf{\%}\end{array}$ & $\begin{array}{c}\mathbf{2 0 - 5 0} \\
\mathbf{\%}\end{array}$ & $\begin{array}{c}</=\mathbf{2 5 0} \\
\mathbf{n g} / \mathbf{m l}\end{array}$ & $\begin{array}{c}<\mathbf{1 0} \\
\mathbf{n g} / \mathbf{m l}\end{array}$ & $\begin{array}{c}\mathbf{4 4 - 9 7} \\
\mathbf{u m o l} / \mathbf{l}\end{array}$ & $\begin{array}{c}\mathbf{1 2 8 - 1 5 0} \\
\mathbf{m m o l} / \mathbf{l}\end{array}$ & $\begin{array}{c}\mathbf{3 . 5}-\mathbf{5 . 5} \\
\mathbf{m m o l} / \mathbf{l}\end{array}$ & $\begin{array}{c}\mathbf{1 0 9 - 1 2 2} \\
\mathbf{m m o l} / \mathbf{l}\end{array}$ \\
\hline $27 \mathrm{Nov}$ & 7.2 & 58.6 & 36.1 & 158 & 12.2 & 76.4 & 132.2 & 3.25 & 108.6 \\
\hline $07 \mathrm{Dec}$ & 7.3 & 54.4 & 41.3 & 1797 & 69.51 & 67.6 & 131.6 & 3.30 & 108.8 \\
\hline 14 Dec & 11.2 & 52.1 & 41.4 & 580.5 & $<2.5$ & 89.6 & 136.4 & 3.24 & 107.7 \\
\hline
\end{tabular}

Table1: Trend of Lab Test Results through the Course of Illness

WBC-Leukocytes, N\%-Proportion of Neutrophil, L\%-Lymphocyte Proportion, Creat-Creatinine, NA - Serum Sodium, $K$-Serum Potassium, Cl - Serum Chloride,CRP- C-Reactive Protein

I commenced him on cerebroprotein at $90 \mathrm{mg}$ once a day, and Nervoplex (a complex with methyl cobalamin and alpha lipoic acid) to hasten the recovery of the nerve, and he displayed asubjective improvement of about $70 \%$ over a period of 3 months.

As seen on Fig. 1, His Xray of the Lumbar-Sacral Spine from both anteroposterior and lateral views were unremarkable and he could not afford to have an MRI done.

His overall improvement by the end of 12 weeks was markedly felt through a marked improvement on the footdrop gait, his improved ability to put on shoes of his correct size without them falling off and ability to stand up from a squatting position without losing balance which he had difficulties with during the onset of the condition about 2 months from his initial presentation, though there is still weakness on the right foot (3/5) compared to the left side (5/5).

\section{Discussion}

COVID-19 has been found to cause encephalitis, olfactory mononeuropathy, and GuillainBarre Syndrome (Montalvan et al., 2020). It also has been found to induce very different presentations due to its unique capability to produce inflammation that effects multiple levels of cytokines. This enables the presentation of COVID-19 to vary in comparison to other viral infections.(Abdelnour et al., 2020)

A flat foot is a very uncomfortable and scary condition to undergo, as it reduces functionality and turns the affected patients gait into a conspicuous presentation that also makes it hard to go to gatherings due to. The thud one walks with is noticed even from a distance, and the worry in most cases is that the condition is not fully reversible. Sensation is not always affected and the L5 radiculopathy can be differentiated from a common peroneal nerve causing a foot drop through the eversion being weakened in a peroneal neuropathy versus and inversion being weakened by the L5 neuropathy. (Graham, 2010)

Cases of foot drop have been only found with mechanical injury to the nerve, with only a few cases presenting due to cardiovascular accidents and sometimes multiple sclerosis. In these cases, it does not cause a mononeuropathy.(Stewart, 2008), (Poage et al., 2016)

With all the above considerations, and the fact that this case was of a man who had no other previous neuropathic conditions or autoimmune conditions. Also, the fact that the onset of this foot drop was acute similar to a vascular event, and it improved with time made it more like a vascular event. Probably similar to an occlusion of the vascular plexus that would supply a specific nerve, if this was possible. The common peroneal nerve is more prone than its neighboring tibial nerves to vascular damage due to its anatomy as well.(Kadiyala et al., 2005)This could lead to a mononeuropathy such as found in this case.

It's interesting that this presentation coincided with his d-dimers reducing on rivaroxabanand aspirin after its initial surge, while he was better clinically from his respiratory symptoms and after he had already turned PCR negative from his nasopharyngeal PCR. It is also interesting that such mononeuropathies have only been affecting the olfactory 
nerve in other cases reported so far. This is the first time the common peroneal nerve was affected.

There was no specific pressure or injury applicable to the right leg or hip. Pressure was found to be the cause of a bilateral common peroneal nerve palsy in a case reported to have anorexia with excessive weight loss leading to bony compression of the nerve fibers along their path bilaterally. (Kershenbaum et al., 1996)

The Xray was clear, and the symptoms improved over time once the CRP and D-dimers had returned completely back to normal. The neuropraxia had no other metabolic cause found, nor a history of mechanical injury in any way to support it. Also, the fact that it was bilateral made it even less likely for it to be mechanical in nature without the patient realizing the injury he would have experienced.

\section{Conclusion}

COVID-19 was initially thought to be an illness with a primarily respiratory focus but as time continues to pass, cases are revealing a number of neurological symptoms in addition to the possibility of other multiple systemic effects of the disease(Lai et al., 2020), more commonly includinghypercoagulability and the myocardial damage(Kochi et al., 2020).

Mononeuropathy related to the olfactory nerve is quite common(Li et al., 2020), however there is no case reported showing a mononeuropathy effecting the common peroneal nerve alone from any infective causes before this case. This nerve has only been reported to be damaged after mechanical injury, with a few cases being found after a stroke and some autoimmune conditions such as multiple sclerosis (Graham, 2010). None of these were applicable to this case.

This case proves to be one of the unique manifestations of sequelae from COVID-19 solely due to the unique immune response induced by COVID-19 infection. The fact that the severity of the symptoms improved with time and hopefully may resolve fully is a reassuring aspect of the presentation of a neuropraxia rather than a long-term neuropathy.

\section{Acknowledgement}

To the patient for giving consent for this publication.

\section{References}

i. Abdelnour, L., EltahirAbdalla, M., \&Babiker, S. (2020). COVID 19 infection presenting as motor peripheral neuropathy. In Journal of the Formosan Medical Association. https://doi.org/10.1016/j.jfma.2020.04.024

ii. Ag Lamat, M. S. N., Khoo, C. S., \&Shaaya, F. (2020). Bilateral foot drop in a young woman. ActaNeurologicaBelgica, 120(6), 1437-1438. https://doi.org/10.1007/s13760-020-01438-8

iii. Graham, J. (2010). Foot drop: Explaining the causes, characteristics and treatment. British Journal of Neuroscience Nursing. https://doi.org/10.12968/bjnn.2010.6.4.47792

iv. Kadiyala, R. K., Ramirez, A., Taylor, A. E., Saltzman, C. L., \&Cassell, M. D. (2005). The blood supply of the common peroneal nerve in the popliteal fossa. Journal of Bone and Joint Surgery - Series B. https://doi.org/10.1302/0301-620X.87B3.15694

v. Kershenbaum, A., Jaffa, T., Zeman, A., \& Boniface, S. (1996). Bilateral Foot-Drop in a Patient with Anorexia Nervosa. 3-5.

vi. Kirschenbaum, D., Imbach, L. L., Ulrich, S., Rushing, E. J., Keller, E., Reimann, R. R., Frauenknecht, K. B. M., Lichtblau, M., Witt, M., Hummel, T., Steiger, P., Aguzzi, A., \&Frontzek, K. (2020). Inflammatory olfactory neuropathy in two patients with COVID-19. In The Lancet. https://doi.org/10.1016/S0140-6736(20)31525-7

vii. Kochi, A. N., Tagliari, A. P., Forleo, G. B., Fassini, G. M., \&Tondo, C. (2020). Cardiac and arrhythmic complications in patients with COVID-19. In Journal of Cardiovascular Electrophysiology. https://doi.org/10.1111/jce.14479

viii. Lai, C. C., Ko, W. C., Lee, P. I., Jean, S. S., \& Hsueh, P. R. (2020). Extra-respiratory manifestations of COVID-19. International Journal of Antimicrobial Agents. https://doi.org/10.1016/j.ijantimicag.2020.106024

ix. Li, C. W., Syue, L. S., Tsai, Y. S., Li, M. C., Lo, C. L., Tsai, C. S., Chen, P. L., Ko, W. C., \& Lee, N. Y. (2020). Anosmia and olfactory tract neuropathy in a case of COVID-19. Journal of Microbiology, Immunology and Infection. https://doi.org/10.1016/j.jmii.2020.05.017

x. Montalvan, V., Lee, J., Bueso, T., De Toledo, J., \& Rivas, K. (2020). Neurological manifestations of COVID-19 and other coronavirus infections: A systematic review. In Clinical Neurology and Neurosurgery. https://doi.org/10.1016/j.clineuro.2020.105921

xi. Poage, C., Roth, C., \& Scott, B. (2016). Peroneal Nerve Palsy: Evaluation and Management. In Journal of the American Academy of Orthopaedic Surgeons. https://doi.org/10.5435/JAAOS-D-14-00420

xii. Stewart, J. D. (2008). Foot drop: Where, why and what to do? In Practical Neurology. https://doi.org/10.1136/jnnp.2008.149393 